\title{
MicroRNA-9-5p increases the sensitivity of colorectal cancer cells to 5-fluorouracil by downregulating high mobility group A2 expression
}

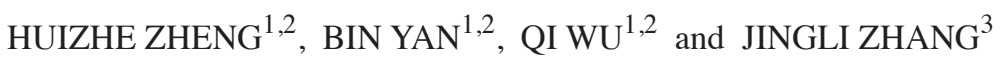 \\ ${ }^{1}$ Department of Pathology, Hongqi Hospital Affiliated to Mudanjiang Medical University; \\ ${ }^{2}$ Key Laboratory of Tumor Prevention and Treatment of Heilongjiang Province, \\ Mudanjiang Medical University; ${ }^{3}$ Department of Rheumatology and Immunology, \\ Hongqi Hospital Affiliated to Mudanjiang Medical University, Mudanjiang, Heilongjiang 157011, P.R. China
}

Received August 11, 2020; Accepted November 26, 2020

DOI: $10.3892 / \mathrm{ol} .2021 .12496$

\begin{abstract}
Chemotherapy drug 5-fluorouracil (5-FU) is the first-line treatment for colorectal cancer (CRC); however, 5-FU resistance decreases CRC therapeutic efficiency. A previous study revealed that microRNA (miR)-9-5p serves an antitumor effect in CRC. However, the effect of miR-9-5p in CRC chemoresistance remains unknown. In the present study, two CRC cell lines, including HT-29 and HCT-116 cells, were used to investigate the impact of miR-9-5p in overcoming 5 -FU resistance. The results revealed that treatment with 5-FU decreased CRC cell viability and upregulated miR-9-5p expression in both CRC cells. Knockdown of miR-9-5p decreased HCT-116 cell sensitivity to 5-FU and inhibited apoptosis. By contrast, miR-9-5p overexpression enhanced the sensitivity of HT-29 cells to 5-FU and induced apoptosis. Additionally, it was confirmed that miR-9-5p directly targeted high mobility group A2 (HMGA2). HMGA2 overexpression reversed miR-9-5p-induced HT-29 apoptosis. The present study indicated that miR-9-5p enhanced the sensitivity of CRC cells to 5-FU via downregulating HMGA2 expression.
\end{abstract}

\section{Introduction}

Colorectal cancer (CRC) is the third most frequent malignant tumor, with 1.85 million new cases per year, and the second primary cause of death worldwide, with 881,000 deaths estimated in 2018 (1). 5-fluorouracil (5-FU) has been widely used as a chemotherapeutic agent for CRC (2). However, the

Correspondence to: Dr Jingli Zhang, Department of Rheumatology and Immunology, Hongqi Hospital Affiliated to Mudanjiang Medical University, 5 Tongxiang Road, Mudanjiang, Heilongjiang 157011, P.R. China

E-mail: zj1116027@126.com

Key words: colorectal cancer, 5-fluorouracil resistance, microRNA-9-5p, high mobility group A2, apoptosis therapeutic effects of 5-FU on patients with CRC are very limited due to the development of drug resistance (3). It is imperative to investigate the potential mechanisms of CRC chemoresistance, which may help to develop novel treatment strategies to improve patient outcomes.

MicroRNAs (miRNAs/miRs), a class of short endogenous non-coding RNAs, serve key roles in gene regulation after transcription via targeting the 3'-untranslated region (UTR) of mRNAs (4). A previous study has revealed the antitumor effects of miRNAs in numerous types of cancer, such as chronic lymphocytic leukemia, prostate cancer and gastrointestinal stromal tumors (5). Dysregulation of miRNAs has been reported to exert a crucial function in drug chemoresistance (6). For example, miR-20a overexpression sensitizes breast cancer cells to chemotherapy drugs and inhibits their proliferation (7). Wang et al (8) demonstrated that miR-26b was involved in 5-FU-induced apoptosis in CRC. Recent studies have suggested that miR-9 behaves as a tumor suppressor in different types of cancer, such as hepatocellular carcinoma (9), gastric cancer (10), acute myeloid leukemia (11) and CRC (12). Cekaite et al (13) observed low miR-9-5p expression in CRC cells and found that miR-9-5p overexpression inhibits cell survival and promotes apoptosis. Additionally, miR-9-5p represses CRC cell migration and invasion via downregulating transmembrane-4-L6 family 1 expression (14). However, the impact of miR-9-5p in CRC chemoresistance remains unclear.

The high mobility group A2 (HMGA2) gene is located on human chromosome 12q14 (15). Accumulating evidence has revealed that HMGA2 is highly expressed in a number of malignant tumors, including lung cancer (16), breast cancer (17) and CRC (18). Previous studies have demonstrated that high HMGA2 expression is associated with a poor prognosis and a lower survival rate in patients with CRC $(18,19)$. Furthermore, $\mathrm{Xu}$ et al (20) revealed that HMGA2 contributes to 5-FU chemoresistance in $\mathrm{CRC}$ via the dishevelled segment polarity protein $2 / \mathrm{Wnt}$ signaling pathway. Based on the aforementioned studies, the present study speculated that miR-9-5p may modulate 5-FU resistance in CRC by regulating HMGA2 expression. 
In the current study, the function and underlying mechanism of miR-9-5p in 5-FU resistance in two CRC cell lines, including HCT-116 and HT-29 cells, were investigated.

\section{Materials and methods}

Cell culture. According to previous studies $(21,22)$, human CRC HCT-116 and HT-29 cell lines were chosen to evaluate the effect of miR-9-5p in CRC chemoresistance. HCT-116 and HT-29 cells were obtained from Procell Life Science \& Technology Co., Ltd., and maintained in McCoy's 5A medium (Procell Life Science \& Technology Co., Ltd.) containing $10 \%$ fetal bovine serum (FBS). 293T cells (Shanghai Zhongqiao Xinzhou Biotechnology Co., Ltd.) were incubated in Dulbecco's modified Eagle's medium (DMEM; Sigma-Aldrich; Merck KGaA) containing 10\% FBS. Cells were cultured at $37^{\circ} \mathrm{C}$ with $5 \% \mathrm{CO}_{2}$.

Cell transfection. Human miR-9-5p agomir (sense, 5'-UCU UUGGUUAUCUAGCUGUAUGA-3' and antisense, 5'-AUA CAGCUAGAUAACCAAAGAUU-3') and its scrambled negative control (NC; sense, 5'-UUCUCCGAACGUGUC ACGUTT-3' and antisense, 5'-ACGUGACACGUUCGG AGAATT-3'), miR-9-5p antagomir (5'-UCAUACAGCUAG AUAACCAAAGA-3') and its scrambled NC (5'-CAGUAC UUUUGUGUAGUACAA-3') were bought from Shanghai GenePharma Co., Ltd. HCT-116 and HT-29 cells were seeded in a 12 -well plate $\left(4 \times 10^{4}\right.$ cells/well $)$ and incubated overnight at $37^{\circ} \mathrm{C}$ with $5 \% \mathrm{CO}_{2}$. At $70 \%$ confluency, HT-29 cells were transfected with 100 pmol miR-9-5p agomir or agomir NC at room temperature for $48 \mathrm{~h}$ using Lipofectamine ${ }^{\circledR} 2000$ (Invitrogen; Thermo Fisher Scientific, Inc.) following the manufacturer's protocol. Similarly, HCT-116 cells were transfected with 100 pmol miR-9-5p antagomir or antagomir NC. An HMGA2-overexpression pcDNA3.1 plasmid (over-HMGA2) was obtained from GenScript, whereas an empty pcDNA3.1 vector (GenScript) was applied as a NC (vector). A small interference (si)RNA sequence of HMGA2 (si-HMGA2; sense, 5'-AGAGGCAGACCUAGGAAAUTT-3' and antisense, 5'-ATTTCCTAGGTCTGCCTCTTT-3') was bought from JTS Scientific, Ltd. A scrambled siRNA (JTS Scientific, Ltd.) was used as a NC (siRNA NC; sense, 5'-UUCUCCGAACGUGUC ACGUTT-3' and antisense, 5'-ACGUGACACGUUCGGAGA ATT-3'). A total of $1 \mu \mathrm{g}$ of over-HMGA 2 was transfected alone or co-transfected with 50 pmol miR-9-5p agomir into HT-29 cells at room temperature for $48 \mathrm{~h}$ using Lipofectamine 2000 following the manufacturer's protocol. HCT-116 cells were transfected with 50 pmol si-HMGA2 or co-transfected with 50 pmol miR-9-5p antagomir and si-HMGA2. Transfected cells were collected $48 \mathrm{~h}$ after incubation for subsequent experiments.

Hoechst staining. After fixation with $4 \%$ paraformaldehyde for $20 \mathrm{~min}$ at room temperature, two CRC cell lines (HCT-116 and HT-29 cells) were used to observe nuclear changes and apoptotic body formation using the Hoechst staining kit (Nanjing KeyGen Biotech Co., Ltd.) according to the manufacturer's protocol. Cells were visualized under a fluorescence microscope (IX53; Olympus Corporation; magnification, $\mathrm{x} 400$ ) and counted by a professional researcher, who was blind to the grouping. The percentage of apoptotic cells was calculated as follows: Apoptotic cells $(\%)=($ the number of apoptotic cells/the number of total cells) $\mathrm{x} 100 \%$.

Reverse transcription-quantitative PCR (RT-qPCR). Total RNA from HCT-116 and HT-29 cells was isolated using the TRIpure lysis buffer (BioTeke Corporation). Subsequently, RT to cDNA was performed using M-MLV Reverse Transcriptase kit (cat. no. PR6502; BioTeke Corporation) according to the manufacturer's protocol. qPCR reactions were performed using the SYBR Green PCR kit (Sigma-Aldrich; Merck KGaA) according to the manufacturer's protocol. The PCR reaction was run for an initial denaturation for $5 \mathrm{~min}$ at $94^{\circ} \mathrm{C}$, followed by 40 cycles of denaturation at $94^{\circ} \mathrm{C}$ for $15 \mathrm{sec}$, annealing at $60^{\circ} \mathrm{C}$ for $25 \mathrm{sec}$ and extension at $72^{\circ} \mathrm{C}$ for $30 \mathrm{sec}$. Subsequently, the $2^{-\Delta \Delta \mathrm{Cq}}$ comparative method was applied to determine the relative expression levels of mRNA and miRNA (23). U6 was used as the internal reference for miR-9-5p, while $\beta$-actin acted as the internal reference for HMGA2. The primer sequences used are listed in Table I.

Dual-luciferase reporter assay. TargetScan (http://www. targetscan.org) was used to predict the potential target genes of miR-9-5p. For the miR-9-5p-target analysis, a fragment of the HMGA2 3'-UTR containing either the wild-type (WT) or mutated (Mut) binding sites for miR-9-5p was cloned in a pmirGLO luciferase reporter vector (Promega Corporation) following the manufacturer's instructions. 293T cells were plated into 12 -well plates and co-transfected using Lipofectamine 2000 with luciferase reporter plasmid and miR-9-5p agomir and agomir NC. After $4 \mathrm{~h}$, cells were incubated in new DMEM for $48 \mathrm{~h}$ at $37^{\circ} \mathrm{C}$. Subsequently, luciferase activities were detected using the Dual-Luciferase Reporter Assay kit (Promega Corporation) following the manufacturer's protocol. Luciferase activity was normalized to the Renilla luciferase activity.

Western blotting. Cells were lysed using the RIPA lysis buffer (Beyotime Institute of Biotechnology) and the supernatants were collected via centrifugation $(10,000 \mathrm{x}$ g for $5 \mathrm{~min}$ at $\left.4^{\circ} \mathrm{C}\right)$. Protein concentration was measured using the BCA Protein Concentration Determination kit (Beyotime Institute of Biotechnology) following the manufacturer's protocol. Protein separation ( $40 \mu \mathrm{g} /$ lane) was performed via 12 or $15 \%$ SDS-PAGE (Beyotime Institute of Biotechnology). Proteins were transferred onto polyvinylidene fluoride membranes (Thermo Fisher Scientific, Inc.) and blocked with 5\% (m/v) skimmed milk, followed by incubation with the following primary antibodies at $4^{\circ} \mathrm{C}$ overnight: Polyclonal rabbit anti-HMGA2 (1:1,000; cat. no. 20795-1-AP; ProteinTech Group, Inc.) and monoclonal mouse anti- $\beta$-actin $(1: 1,000$; cat. no. sc-47778; Santa Cruz Biotechnology, Inc.). After washing with TBS-Tween $(0.1 \%$ Tween-20), proteins were incubated with HRP-conjugated goat anti-rabbit $\operatorname{IgG}$ (1:5,000; cat. no. A0208; Beyotime Institute of Biotechnology) and goat anti-mouse IgG (1:5,000; cat. no. A0216; Beyotime Institute of Biotechnology) secondary antibodies for $40 \mathrm{~min}$ at $37^{\circ} \mathrm{C}$. The protein bands were visualized using an enhanced chemiluminescence kit (Beyotime Institute of Biotechnology) according to the manufacturer's protocol, and quantified 
Table I. Primers used for reverse transcription-quantitative PCR.

\begin{tabular}{ll} 
Gene & \multicolumn{1}{c}{ Sequence (5'-3') } \\
\hline HMGA2 & F: CTTCAGCCCAGGGACAAC \\
& R: TCCAGTGGCTTCTGCTTTC \\
3-actin & F: CTTAGTTGCGTTACACCCTTTCTTG \\
U6 & R: CTGTCACCTTCACCGTTCCAGTTT \\
& F: GCTTCGGCAGCACATATACT \\
hsa-miR-9-5p & F: GTGCAGGGTCCGAGGTATTC \\
& R: GCAGCCTCTTTGGTTATCTAGC \\
&
\end{tabular}

HMGA2, high mobility group A2; miR, microRNA; F, forward; R, reverse.

using the Gel-Pro-Analyzer 4.0 software (Media Cybernetics, Inc.).

Cell counting Kit-8 (CCK-8) assay. Briefly, 4x103 cells/well were plated in a 96-well plate. Subsequently, CRC cells were subjected to distilled water (vehicle control group) or different concentrations of 5-FU $(0.625,1.25,2.5,5,10,20,40$ and $80 \mu \mathrm{M}$; Shanghai Aladdin Biochemical Technology Co., Ltd.). After $48 \mathrm{~h}$ at $37^{\circ} \mathrm{C}$, the CCK-8 assay kit (Beyotime Institute of Biotechnology) was used to measure $\mathrm{CRC}$ cell viability at $37^{\circ} \mathrm{C}$ for $1 \mathrm{~h}$ following the manufacturer's protocol. Optical density values at $450 \mathrm{~nm}$ were detected using an ELX-800 microplate reader (BioTek Instruments, Inc.; Agilent Technologies, Inc.).

Apoptosis assay. The Annexin V-FITC Apoptosis Detection kit (Nanjing KeyGen Biotech Co., Ltd.) was used to measure apoptosis. After washing two times with PBS, CRC cells were double-stained with Annexin V-FITC and PI in the dark for $10 \mathrm{~min}$ at room temperature. Transfected cells were subjected to 2.5 or $10 \mu \mathrm{M} 5$-FU. After $48 \mathrm{~h}$, apoptosis was measured via flow cytometry (NovoCyte; ACEA Biosciences, Inc.; Agilent Technologies, Inc.) and analyzed using NovoExpress 1.2.5 (ACEA Biosciences, Inc.; Agilent Technologies, Inc.).

Caspase activity assay. Cells were lysed in cold lysis buffer and maintained on ice for $15 \mathrm{~min}$. After centrifugation at $16,000 \mathrm{x} \mathrm{g}$ for $15 \mathrm{~min}$ at $4^{\circ} \mathrm{C}$, protein concentration in the cell supernatant was detected using the Bradford Protein Concentration Determination kit (Beyotime Institute of Biotechnology). Subsequently, caspase-3 and activities of the CRC cells were measured using the Caspase 3 Activity Assay kit (cat. no. C1116; Beyotime Institute of Biotechnology) and caspase-9 activities were detected using the Caspase 9 Activity Assay kit (cat. no. BC3890; Beijing Solarbio Science \& Technology Co., Ltd.), following the manufacturers' protocol.

Statistical analysis. Statistical analysis was performed using GraphPad Prism (v8.0; GraphPad Software, Inc.) and data were presented as the mean $\pm \mathrm{SD}(\mathrm{n}=3)$. $\mathrm{IC}_{50}$ values were measured using GraphPad Prism. The $\mathrm{IC}_{50}$ value represents the drug concentration at which tumor cells are inhibited by half. Unpaired two-tailed Student's t-test was used for comparisons between two groups, while one-way or two-way ANOVA with Tukey's post-hoc test was used for multiple comparisons to analyze statistically significant differences. $\mathrm{P}<0.05$ was considered to indicate a statistically significant difference.

\section{Results}

5-FU upregulates miR-9-5p expression in CRC cells. The effect of 5-FU on two CRC cell lines, including HT-29 and HCT-116 cells, was assessed via CCK-8 assay. The cell viability of both CRC cell lines was significantly decreased in a concentration-dependent $(0.65-80 \mu \mathrm{M})$ manner compared with the vehicle group $(0 \mu \mathrm{M} 5-\mathrm{FU})$ (Fig. $1 \mathrm{~A} ; \mathrm{P}<0.05)$. HCT-116 cells were more sensitive to 5-FU than HT-29 cells $\left(\mathrm{IC}_{50} 6.343 \pm 0.767\right.$ and $30.510 \pm 3.440 \mu \mathrm{M}$, respectively). A previous study demonstrated that miR-9-5p expression was markedly lower in CRC cells compared with that in normal colorectal epithelial cells (13); thus, the present study further assessed miR-9-5p expression in 5-FU-treated CRC cells. The RT-qPCR results revealed that treatment with 5-FU significantly upregulated miR-9-5p expression in both CRC cells in a dose-dependent manner compared with the vehicle group (Fig. $1 \mathrm{~B} ; \mathrm{P}<0.05$ ).

miR-9-5p overexpression enhances $C R C$ cell sensitivity to $5-F U$. To explore the effect of miR-9-5p in overcoming 5-FU resistance, HT-29 cells with a high $\mathrm{IC}_{50}$ value were transfected with miR-9-5p agomir or agomir NC, and HCT-116 cells with a low $\mathrm{IC}_{50}$ value were transfected with miR-9-5p antagomir or antagomir NC. The results revealed that miR-9-5p overexpression significantly increased miR-9-5p expression in HT-29 cells, while miR-9-5p expression was significantly downregulated in HCT-116 transfected with antagomiR-9-5p (Fig. 2A; $\mathrm{P}<0.05)$. The CCK-8 analysis indicated that after transfection with miR-9-5p agomirs and antagomirs, treatment with 5-FU significantly decreased CRC cell viability in a dose-dependent manner (Fig. 2B; P<0.05). Upregulation of miR-9-5p significantly enhanced HT-29 cell sensitivity to 5-FU, with the $\mathrm{IC}_{50}$ value decreasing from $28.488 \pm 4.069 \mu \mathrm{M}$ in the agomir $\mathrm{NC}$ group to $15.680 \pm 2.001 \mu \mathrm{M}$ in the miR-9-5p agomir group, whereas miR-9-5p-knockdown decreased HCT-116 cell sensitivity to $5-\mathrm{FU}$, with the $\mathrm{IC}_{50}$ value increasing from $6.464 \pm 0.686$ to $17.586 \pm 2.501 \mu \mathrm{M}$ in the antagomir $\mathrm{NC}$ and miR-9-5p antagomir groups, respectively (Fig. 2B; $\mathrm{P}<0.05$ ). Additionally, based on the $\mathrm{IC}_{50}$ value of the two $\mathrm{CRC}$ cell lines (HT-29 and HCT-116), one-third of the $\mathrm{IC}_{50}$ value $(2.5 \mu \mathrm{M}$ 5-FU for HCT-116 cells and $10 \mu \mathrm{M}$ 5-FU for HT-29 cells) was chosen to explore the impact of miR-9-5p on the sensitivity of CRC cells to 5-FU. The results revealed that miR-9-5p overexpression significantly induced HT-29 apoptosis, whereas knockdown of miR-9-5p significantly repressed the apoptosis of HCT-116 cells (Fig. 2C; P<0.05). Hoechst staining revealed that the nuclei of apoptotic cells were densely stained after transfection with the miR-9-5p agomir compared with the agomir NC, while treatment with miR-9-5p antagomir decreased the staining number of apoptotic cells compared with the antagomir NC (Fig. 2D). Corresponding quantitative analysis revealed that the percentage of apoptotic cells was significantly increased by miR-9-5p agomir and significantly decreased by miR-9-5p antagomir compared with the corresponding NCs (Fig. 2D; P<0.05). Furthermore, the expression 

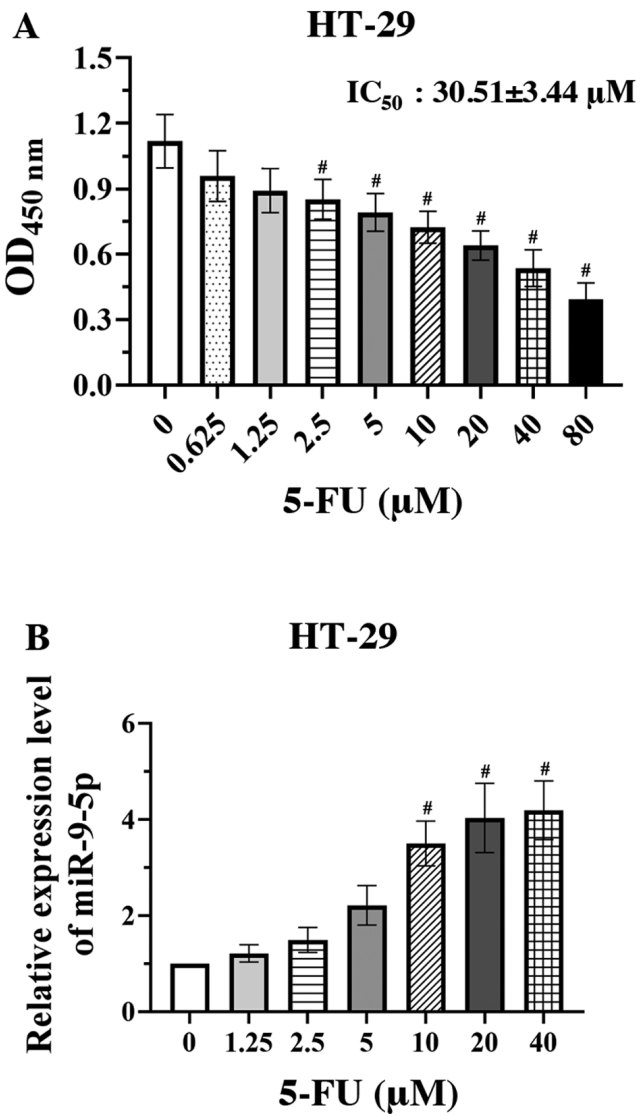

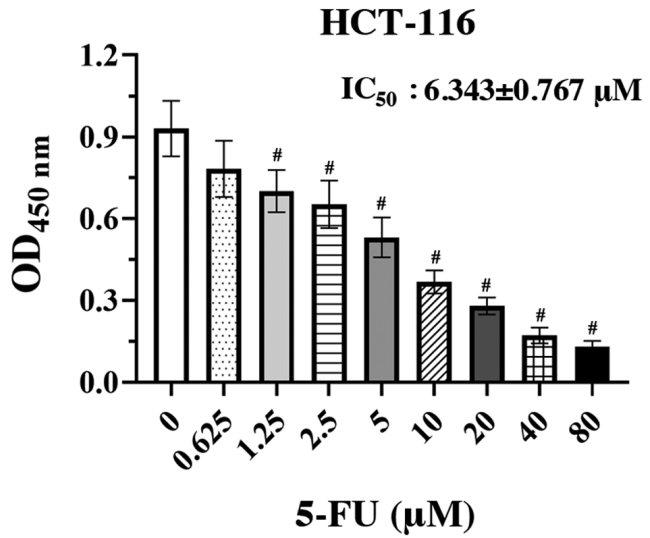

HCT-116

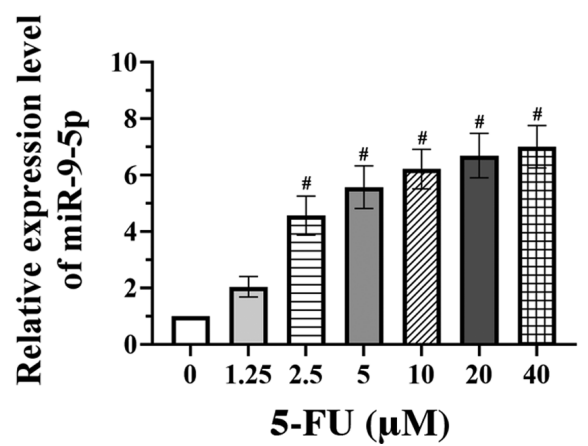

Figure 1. 5-FU upregulates miR-9-5p expression in CRC cells. (A) Cell counting kit-8 analysis was used to measure CRC cell viability. HT-29 and HCT-116 cells were subjected to different concentrations of 5-FU for $48 \mathrm{~h}$. (B) HT-29 and HCT-116 cells were subjected to various concentrations of 5-FU for $48 \mathrm{~h}$, and reverse transcription-quantitative PCR was used to measure the mRNA levels of miR-9-5p. ${ }^{\#} \mathrm{P}<0.05$ vs. the vehicle group ( $\mu \mathrm{M}$ 5-FU). Data are shown as the mean \pm SD $(n=3)$. OD, optical density; 5-FU, 5-fluorouracil; CRC, colorectal cancer; miR, microRNA.

levels of apoptosis-associated markers, including caspase-3 and caspase-9, were significantly upregulated after miR-9-5p overexpression and downregulated after miR-9-5p-knockdown (Fig. 2E; P<0.05).

miR-9-5p directly targets HMGA2. To clarify the functional effect of miR-9-5p in CRC cell sensitivity to 5-FU, bioinformatics analysis was applied to predict its target genes. The results revealed that $\mathrm{HMGA} 2$ was one of target genes of miR-9-5p. Therefore, HMGA2 expression was determined by RT-qPCR and western blot assays. The results indicated that compared with the vehicle group ( $0 \mu \mathrm{M} 5-\mathrm{FU})$, treatment with 5-FU decreased HMGA2 expression in the two CRC cell lines (Fig. 3A and B). Additionally, miR-9-5p overexpression significantly downregulated HMGA2 expression in HT-29 cells, whereas miR-9-5p silencing significantly upregulated HMGA2 expression in HCT-116 cells compared with their respective controls (Fig. $3 \mathrm{~A}$ and $\mathrm{B} ; \mathrm{P}<0.05$ ). TargetScan predicted that miR-9-5p potentially bound to HMGA 2 mRNA. There was a targeted binding association between miR-9-5p and HMGA2 (Fig. 3C). Results of the luciferase reporter assay verified that miR-9-5p overexpression significantly repressed the luciferase activity of the reporter via binding to the WT but not the MUT 3'-UTR of HMGA2 (Fig. 3C; P<0.05).

Overexpression of HMGA2 reverses miR-9-5p-induced HT-29 apoptosis. To confirm whether HMGA2 was involved in miR-9-5p-mediated CRC drug resistance response, $\mathrm{HMGA} 2$-overexpression plasmid was co-transfected with miR-9-5p agomir into HT-29 cells. Additionally, a HMGA2 siRNA was co-transfected with miR-9-5p antagomir into HCT-116 cells. Fig. 4A shows that HMGA2 expression was significantly upregulated in HT-29 cells after transfection with over-HMGA2 and significantly downregulated in si-HMGA2-transfected HCT-116 cells $(\mathrm{P}<0.05)$. After co-transfection with miR-9-5p agomir + vector/over-HMGA2 into HT-29 cells, and miR-9-5p antagomir + siRNA NC/si-HMGA2 into HCT-116 cells, CCK-8 assays revealed that overexpression of HMGA2 significantly increased the viability of miR-9-5p agomir-transfected HT-29 cells, whereas the viability of miR-9-5p antagomir-transfected HCT-116 cells was significantly inhibited by HMGA2-knockdown (Fig. 4B; P<0.05). Results of flow cytometry analysis exhibited that the apoptotic rates in miR-9-5p agomir-transfected HT-29 cells were significantly decreased after HMGA2 overexpression, while increased apoptosis was observed in miR-9-5p antagomir-transfected HCT-116 cells following downregulation of HMGA2 expression (Fig. 4C; $\mathrm{P}<0.05$ ). A similar trend was observed in the caspase activity assays. As shown in Fig. 4D, HMGA2 overexpression significantly decreased the levels of caspase- 3 and caspase-9 in miR-9-5p agomir-transfected HT-29 cells, while HMGA2-knockdown significantly increased the levels of caspase- 3 and caspase- 9 

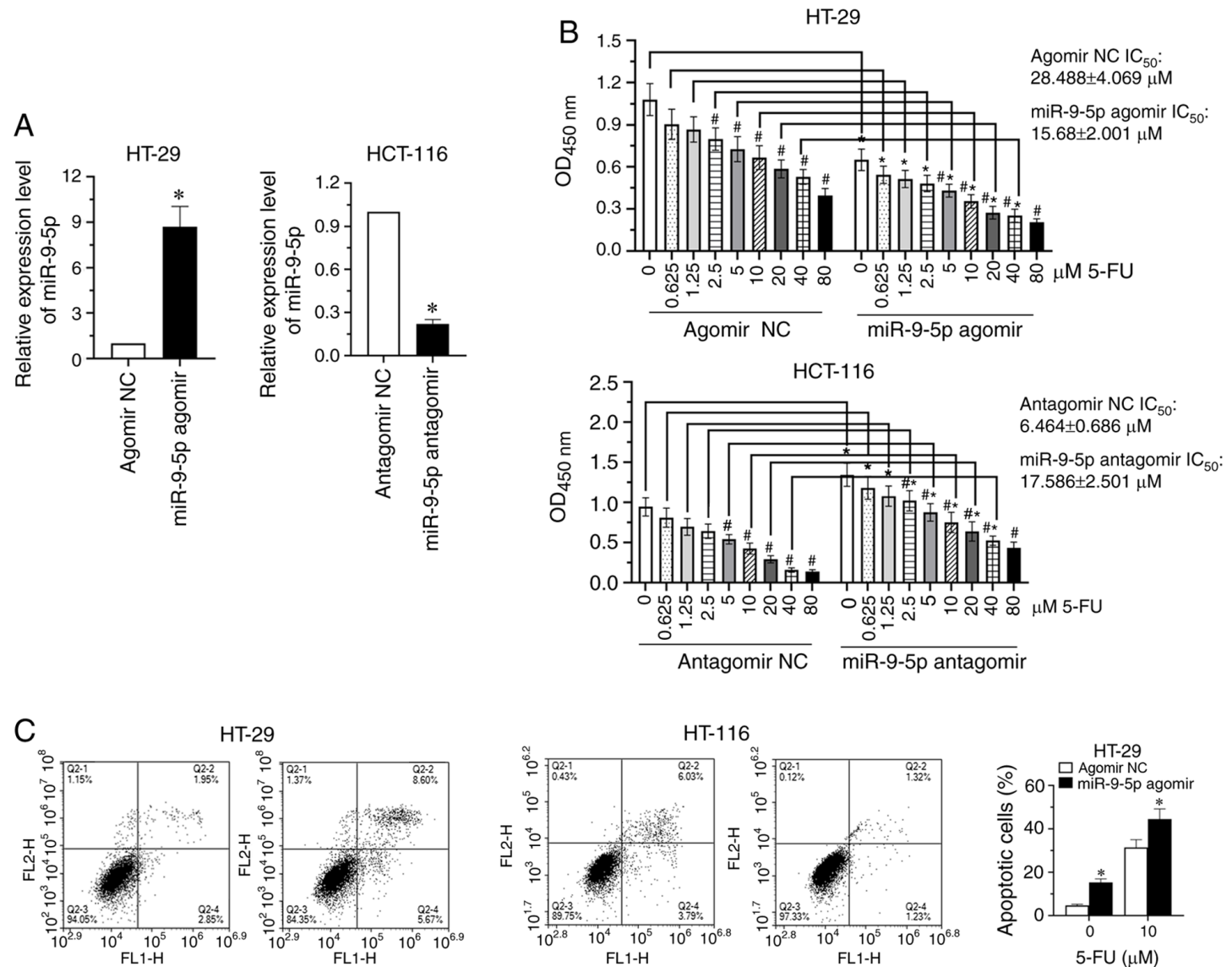

$\mathrm{HT}-116$

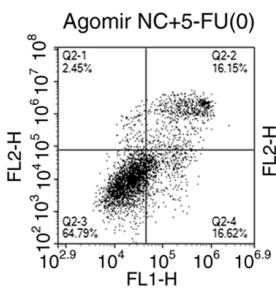

miR-9-5p agomir+5-FU(0)
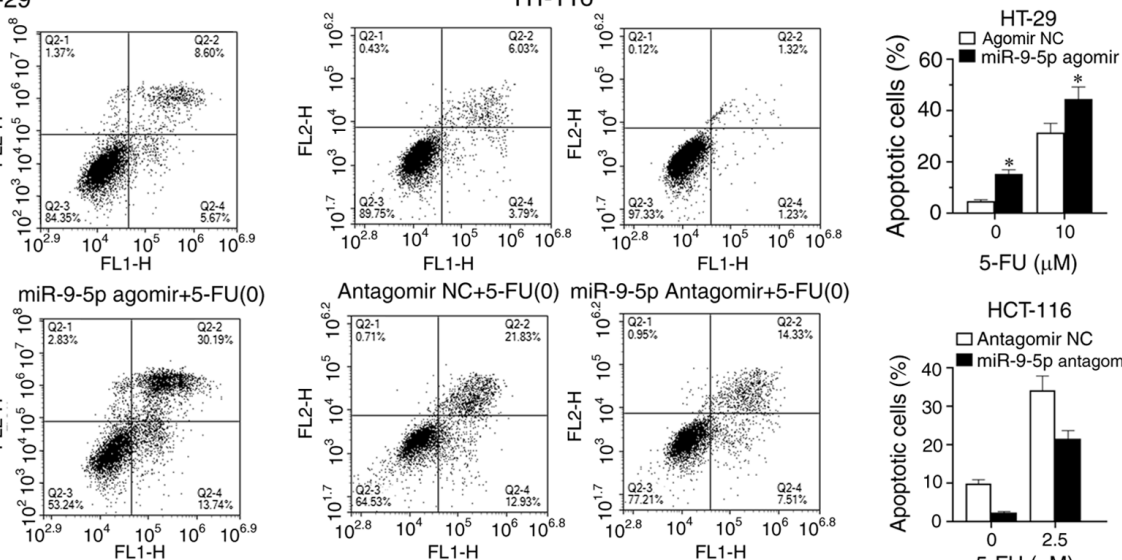

Agomir $\mathrm{NC}+5-\mathrm{FU}(10) \quad$ miR-9-5p agomir+5-FU(10)

Antagomir NC+5-FU(2.5) miR-9-5p antagomir+5-FU(2.5)
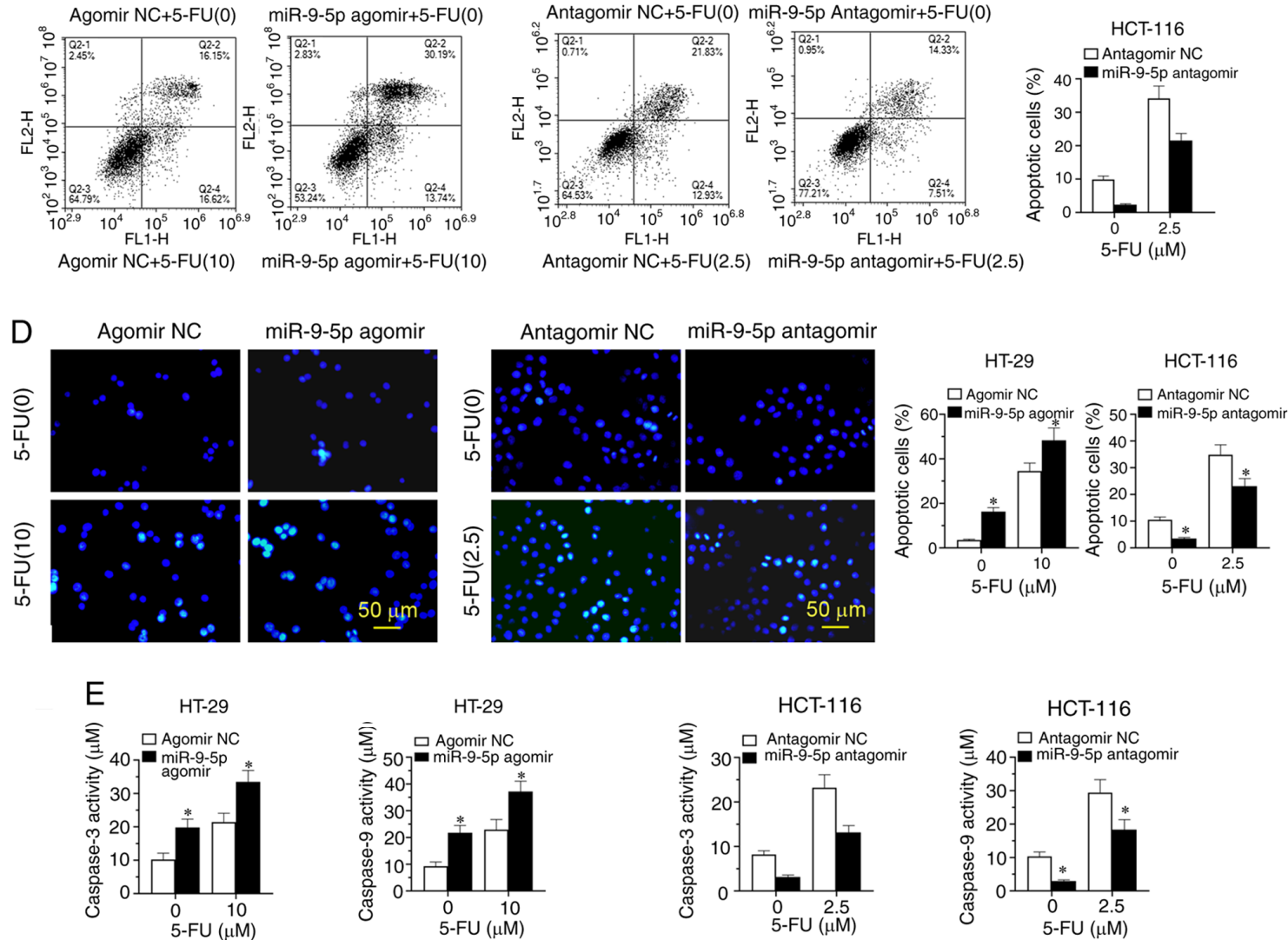

Figure 2. miR-9-5p overexpression enhances CRC cell sensitivity to 5-FU. (A) CRC cells (HT-29 and HCT-116) were transfected with miR-9-5p agomir, antagomir or their respective NCs. After 48 h, the mRNA levels of miR-9-5p were determined by reverse transcription-quantitative PCR. (B) Transfected CRC cells were subjected to different concentrations of 5-FU. After 48 h, cell viability was measured via Cell Counting Kit- 8 assay. (C) Transfected cells were subjected to 2.5 or $10 \mu \mathrm{M} 5$-FU. After $48 \mathrm{~h}$, apoptosis was examined by flow cytometry. (D) Hoechst staining was used to observe the morphological changes. (E) Caspase-3 and caspase- 9 activities were determined $48 \mathrm{~h}$ after transfection. ${ }^{*} \mathrm{P}<0.05$ vs. the vehicle group $\left(0 \mu \mathrm{M} 5\right.$-FU); ${ }^{*} \mathrm{P}<0.05$ vs. the respective agomir/antagomir NC group. Data are shown as the mean \pm SD $(n=3)$. OD, optical density; 5-FU, 5-fluorouracil; CRC, colorectal cancer; miR, microRNA; $\mathrm{NC}$, negative control. 


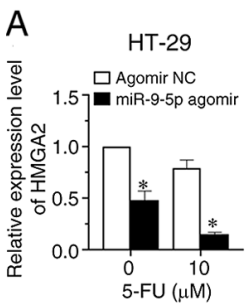

B

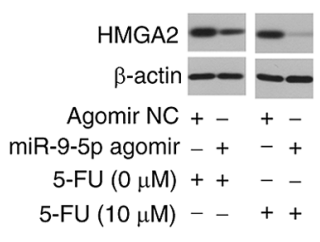

HCT-116

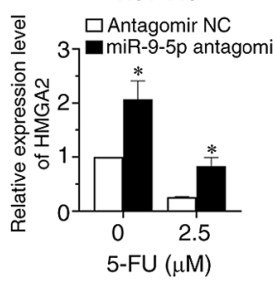

HT-29

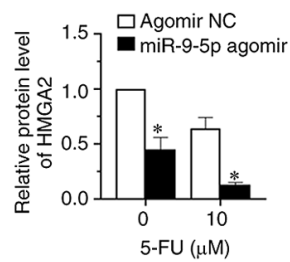

HCT-116

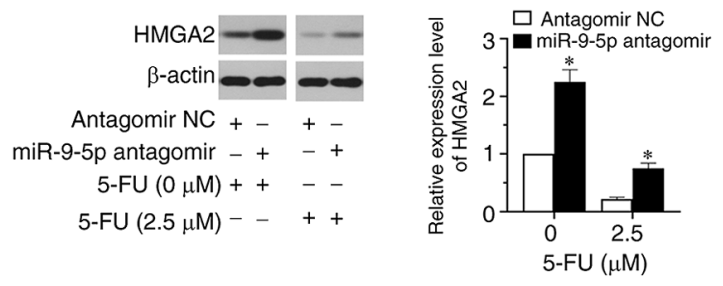

C

Conserved

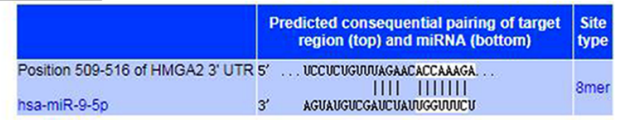

HMGA2-WT 5'-UCCUCUGUUUAGAACACCAAAGA-3 hsa-miR-9-5p 3'-AGUAUGUCGAUCUAUÚGGUUUCUU-5'

HMGA2-Mut 5'-UCCUCUGUUUAGAACUGGUUUCA-3'

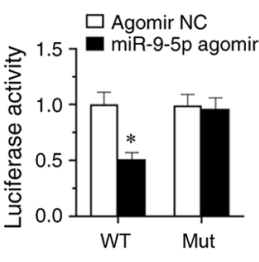

Figure 3. HMGA2 is a target gene of miR-9-5p. (A) Reverse transcription-quantitative PCR and (B) western blotting were used to determine the mRNA and protein levels of HMGA2 in colorectal cancer cells. (C) The target and the binding sites of miR-9-5p were analysed using TargetScan. Luciferase reporter assay was used in HT-29 cells to measure the relative luciferase activities of WT and Mut reporters. ${ }^{*} \mathrm{P}<0.05$ vs. the agomir/antagomir NC groups. Data are shown as the mean $\pm \mathrm{SD}(\mathrm{n}=3)$. WT, wild-type; Mut, mutant; UTR, untranslated region; 5-FU, 5-fluorouracil; miR, microRNA; NC, negative control; HMGA2, high mobility group A2.

A
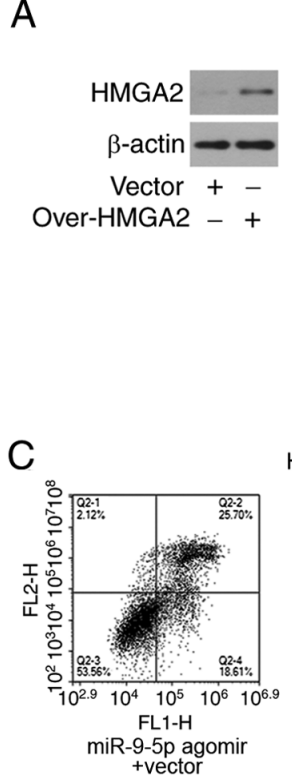
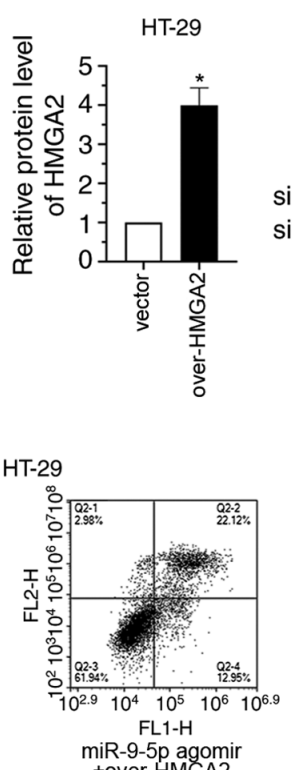

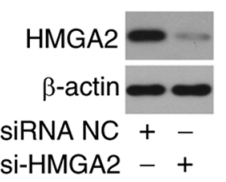

Si-HMGA2 - +
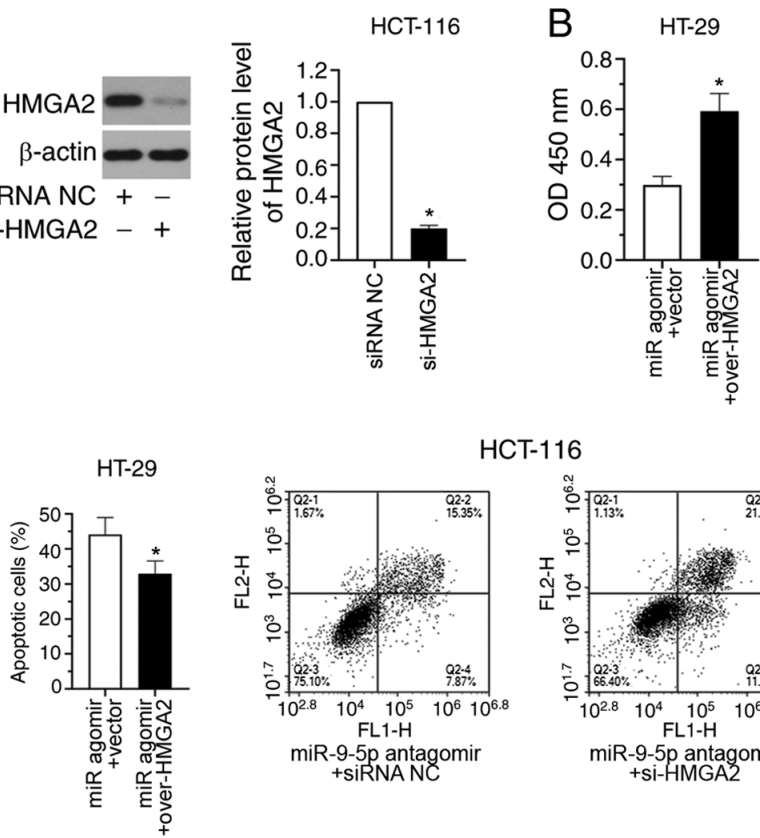

HCT-116
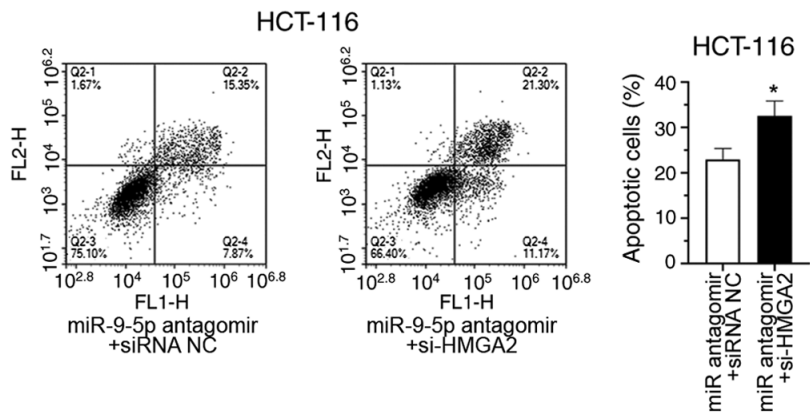
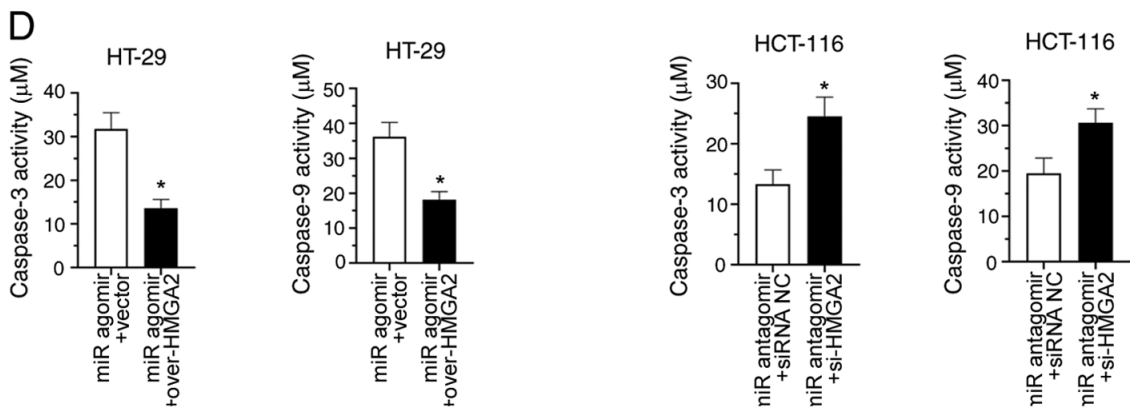

Figure 4. HMGA2 overexpression reverses miR-9-5p-induced HT-29 apoptosis. (A) HT-29 cells were transfected with the HMGA2-overexpression plasmid, while HCT-116 cells were transfected with HMGA2 siRNA. After $48 \mathrm{~h}$, the protein levels of HMGA2 were detected by western blotting. Subsequently, HMGA2-overexpression plasmid was co-transfected with miR-9-5p agomir into HT-29 cells, while HMGA2 siRNA was co-transfected with miR-9-5p antagomir into HCT-116 cells. Transfected cells were subjected to 2.5 or $10 \mu \mathrm{M} 5$-FU. After $48 \mathrm{~h}$ of transfection, (B) cell viability was analysed using the Cell Counting Kit-8 assay, (C) apoptosis was examined by flow cytometry and (D) caspase-3 and caspase- 9 activities were determined. "P<0.05. Data are shown as the mean $\pm \mathrm{SD}(\mathrm{n}=3)$. OD, optical density; 5-FU, 5 -fluorouracil; miR, microRNA; NC, negative control; HMGA2, high mobility group A2; siRNA, small interfering RNA; over, overexpression. 
in miR-9-5p antagomir-transfected HCT-116 cells $(\mathrm{P}<0.05)$. The current findings indicated that HMGA 2 overexpression reversed miR-9-5p-induced apoptosis of HT-29 cells

\section{Discussion}

Various types of treatment plans have been developed for a more effective treatment of CRC; 5-FU is one of the most important anticancer drugs for the therapy of CRC (2). However, resistance to anticancer drugs frequently drives tumor progression $(2,3)$. Accumulating studies have demonstrated that miRNAs serve vital roles in drug resistance for cancer chemotherapy based on their functions and targets $(24,25)$ A previous study revealed that upregulation of miR-9-5p increased the sensitivity of hepatocellular carcinoma cells to cisplatin via modulating epithelial-mesenchymal transition by eukaryotic translation initiation factor 5A2 signaling (9). However, whether miR-9-5p is involved in 5-FU resistance of CRC is unknown. A previous study suggested that miR-9-5p expression was lower in CRC cells, including HCT-116 and HT-29 cells, compared with that in normal colorectal epithelial cells (13). In the current study, the functional effect of miR-9-5p in CRC chemoresistance was further investigated and the targeted signaling that mediated CRC escaping from drug toxicity was explored. The present study revealed that the cell viability of HT-29 cells (p53 wild-type) and HCT-116 cells (TP53 allele homozygous mutant) (26) was decreased in a concentration-dependent manner after 5-FU treatment. HCT-116 cells seemed more sensitive to 5-FU than HT-29 cells. van Boxtel et al (27) have reported that homozygous mutant rats lacking TP53 exhibit decreased survival due to spontaneous tumor development compared with heterozygous rats, which can explain the aforementioned finding. Additionally, RT-qPCR was performed to examine miR-9-5p expression, and the results suggested that 5-FU significantly upregulated miR-9-5p expression in CRC cells. Notably, overexpression of miR-9-5p suppressed cell viability and induced apoptosis in 5-FU-treated HT-29 cells, whereas these responses were mitigated by miR-9-5p-knockdown. Application of miR-9-5p agomir alleviated 5-FU chemoresistance in HT-29 cells. The present study confirmed the crucial role of miR-9-5p in 5-FU chemoresistance.

Numerous studies have demonstrated that miR-9 acts as a tumor suppressor by binding to various targets in CRC, such as C-X-C motif chemokine receptor 4 (12), ubiquitin-like with plant homeodomain and ring finger domains 1 (28) and P21-activated kinase 4 (29). However, the association between miR-9-5p and its target gene in CRC cells to 5-FU resistance has not been defined. The chromatin structuring protein HMGA2 has been found to affect the chemosensitivity of CRC to irinotecan and the stability of human subtelomere (30). A previous study revealed that HMGA2 promotes gemcitabine resistance in pancreatic cancer cells in vitro by increasing histone acetylation (31). Additionally, high HMGA2 expression protects CRC cells from DNA breaks caused by the drug irinotecan, which is a clinically important inhibitor of DNA topoisomerases I (TOP-I) (30). During the shift of replication forks, the transient accumulation of DNA supercoil disrupts gene stability and is manipulated by DNA TOP (30). The protective role of HMGA2 may be due to inhibition of the formation of
TOP-I/DNA complexes (32). On the other hand, low HMGA2 expression contributes to the formation of TOP-I/DNA complexes induced by irinotecan (30). Tsavaris et al (33) have confirmed that TOP-I is highly expressed in CRC following 5-FU treatment. In the current study, miR-9-5p overexpression significantly decreased HMGA2 expression by directly targeting the 3'-UTR of HMGA2. Overexpression of HMGA2 restored miR-9-5p-repressed 5-FU chemoresistance in vitro. The present findings indicated an association between miR-9-5p and 5-FU resistance in CRC. Further exploration is required on whether TOP-I may be a miR-9-5p-inhibited 5-FU sensitivity determinant.

In summary, the present study confirmed that the inhibition of miR-9-5p in 5-FU resistance was mediated by downregulating HMGA2 expression. miR-9-5p enhanced the CRC cell sensitivity to 5-FU and induced apoptosis. The current data provides evidence supporting the association of miR-9-5p with 5-FU resistance in CRC.

\section{Acknowledgements}

Not applicable.

\section{Funding}

The present study was supported by a grant from the Fundamental Research Business Expense of Universities in Heilongjiang Province (grant no. 2018-KYYWFMY-0056).

\section{Availability of data and materials}

The datasets used and/or analyzed during the current study are available from the corresponding author on reasonable request.

\section{Authors' contributions}

HZ and BY designed the study. HZ and QW performed the research and analysed the data. $\mathrm{HZ}$ wrote the first draft of the manuscript. JZ performed the supplementary experiments and revised the manuscript. All authors read and approved the final manuscript.

\section{Ethics approval and consent to participate}

Not applicable.

\section{Patient consent for publication}

Not applicable.

\section{Competing interests}

The authors declare that they have no competing interests.

\section{References}

1. Pisano A, Griñan-Lison C,Farace C,Fiorito G,Fenu G, Jiménez G, Scognamillo F, Peña-Martin J, Naccarati A, Pröll J, et al: The inhibitory role of miR-486-5p on CSC phenotype has diagnostic and prognostic potential in colorectal cancer. Cancers (Basel) 12: $3432,2020$. 
2. Giuliani J and Bonetti A: The pharmacological costs of first-line therapies in unselected patients with advanced colorectal cancer: A review of published phase III trials. Clin Colorectal Cancer 15: 277-284, 2016

3. Yao J, Huang A, Zheng X, Liu T, Lin Z, Zhang S, Yang Q, Zhang T and $\mathrm{Ma} \mathrm{H}$ : 53BP1 loss induces chemoresistance of colorectal cancer cells to 5-fluorouracil by inhibiting the ATM-CHK2-P53 pathway. J Cancer Res Clin Oncol 143: 419-431, 2017.

4. Wu C, Bardes EE, Jegga AG and Aronow BJ: ToppMiR: Ranking microRNAs and their mRNA targets based on biological functions and context. Nucleic Acids Res 42 (Web Server Issue): W107-W113, 2014

5. Berindan-Neagoe I, Monroig Pdel C, Pasculli B and Calin GA: MicroRNAome genome: A treasure for cancer diagnosis and therapy. CA Cancer J Clin 64: 311-336, 2014.

6. Zhao L, Shan Y, Liu B, Li Y and Jia L: Retraction Note: Functional screen analysis reveals miR-3142 as central regulator in chemoresistance and proliferation through activation of the PTEN-AKT pathway in CML. Cell Death Dis 11: 121, 2020

7. Si W, Shen J, Du C, Chen D, Gu X, Li C, Yao M, Pan J, Cheng J, Jiang D, et al: A miR-20a/MAPK1/c-Myc regulatory feedback loop regulates breast carcinogenesis and chemoresistance. Cell Death Differ 25: 406-420, 2018.

8. Wang B, Lu FY, Shi RH, Feng YD, Zhao XD, Lu ZP, Xiao L, Zhou GQ, Qiu JM and Cheng CE: miR-26b regulates 5-FU-resistance in human colorectal cancer via down-regulation of Pgp. Am J Cancer Res 8: 2518-2527, 2018.

9. Bao Y, Zhang Y, Lu Y, Guo H, Dong Z, Chen Q, Zhang X, Shen W, Chen W and Wang X: Overexpression of microRNA-9 enhances cisplatin sensitivity in hepatocellular carcinoma by regulating EIF5A2-mediated epithelial-mesenchymal transition. Int J Biol Sci 16: 827-837, 2020.

10. Meng Q, Xiang L, Fu J, Chu X, Wang C and Yan B: Transcriptome profiling reveals miR-9-3p as a novel tumor suppressor in gastric cancer. Oncotarget 8: 37321-37331, 2017.

11. Zhu B, Xi X, Liu Q, Cheng Y and Yang H: miR-9 functions as a tumor suppressor in acute myeloid leukemia by targeting CX chemokine receptor 4. Am J Transl Res 11: 3384-3397, 2019.

12. Xiong WC, Han N, Ping GF, Zheng PF, Feng HL, Qin L and He P: microRNA-9 functions as a tumor suppressor in colorectal cancer by targeting CXCR4. Int J Clin Exp Pathol 11: 526-536, 2018.

13. Cekaite L, Rantala JK, Bruun J, Guriby M, Agesen TH, Danielsen SA, Lind GE, Nesbakken A, Kallioniemi O, Lothe RA and Skotheim RI: miR-9, -31, and -182 deregulation promote proliferation and tumor cell survival in colon cancer. Neoplasia 14: 868-879, 2012

14. Park YR, Lee ST, Kim SL, Liu YC, Lee MR, Shin JH, Seo SY, Kim SH, Kim IH, Lee SO and Kim SW: MicroRNA-9 suppresses cell migration and invasion through downregulation of TM4SF1 in colorectal cancer. Int J Oncol 48: 2135-2143, 2016.

15. Borrmann L, Schwanbeck R, Heyduk T, Seebeck B, Rogalla P, Bullerdiek J and Wiśniewski JR: High mobility group A2 protein and its derivatives bind a specific region of the promoter of DNA repair gene ERCC1 and modulate its activity. Nucleic Acids Res 31: 6841-6851, 2003.

16. Gao X, Dai M, Li Q, Wang Z, Lu Y and Song Z: HMGA2 regulates lung cancer proliferation and metastasis. Thorac Cancer 8 : 501-510, 2017.

17. Mansoori B, Mohammadi A, Asadzadeh Z, Shirjang S Minouei M, Abedi Gaballu F, Shajari N, Kazemi T, Gjerstorff MF, Duijf PHG and Baradaran B: HMGA2 and Bach-1 cooperate to promote breast cancer cell malignancy. J Cell Physiol 234 17714-17726, 2019.

18. Wang X, Liu X, Li AY, Chen L, Lai L, Lin HH, Hu S, Yao L, Peng J, Loera S, et al: Overexpression of HMGA2 promotes metastasis and impacts survival of colorectal cancers. Clin Cancer Res 17: 2570-2580, 2011.
19. Li Y, Zhao Z, Xu C, Zhou Z, Zhu Z and You T: HMGA2 induces transcription factor Slug expression to promote epithelialto-mesenchymal transition and contributes to colon cancer progression. Cancer Lett 355: 130-140, 2014.

20. Xu X, Wang Y, Deng H, Liu C, Wu J and Lai M: HMGA2 enhances 5-fluorouracil chemoresistance in colorectal cancer via the Dvl2/Wnt pathway. Oncotarget 9: 9963-9974, 2018.

21. Wang YN, Zeng ZL, Lu J, Wang Y, Liu ZX, He MM, Zhao Q, Wang ZX, Li T, Lu YX, et al: CPT1A-mediated fatty acid oxidation promotes colorectal cancer cell metastasis by inhibiting anoikis. Oncogene 37: 6025-6040, 2018.

22. Lesuffleur T, Violette S, Vasile-Pandrea I, Dussaulx E, Barbat A, Muleris M and Zweibaum A: Resistance to high concentrations of methotrexate and 5-fluorouracil of differentiated HT-29 colon-cancer cells is restricted to cells of enterocytic phenotype. Int J Cancer 76: 383-392, 1998.

23. Livak KJ and Schmittgen TD: Analysis of relative gene expression data using real-time quantitative PCR and the 2(-Delta Delta C(T)) method. Methods 25: 402-408, 2001.

24. Longley DB and Johnston PG: Molecular mechanisms of drug resistance. J Pathol 205: 275-292, 2005.

25. Xia H, Ooi LL and Hui KM: MicroRNA-216a/217-induced epithelial-mesenchymal transition targets PTEN and SMAD7 to promote drug resistance and recurrence of liver cancer. Hepatology 58: 629-641, 2013.

26. Ahmed D, Eide PW, Eilertsen IA, Danielsen SA, Eknæs M, Hektoen M, Lind GE and Lothe RA: Epigenetic and genetic features of 24 colon cancer cell lines. Oncogenesis 2: e71, 2013.

27. van Boxtel R, Kuiper RV, Toonen PW, van Heesch S, Hermsen R, de Bruin A and Cuppen E: Homozygous and heterozygous p53 knockout rats develop metastasizing sarcomas with high frequency. Am J Pathol 179: 1616-1622, 2011.

28. Zhu M, Xu Y, Ge M, Gui Z and Yan F: Regulation of UHRF1 by microRNA-9 modulates colorectal cancer cell proliferation and apoptosis. Cancer Sci 106: 833-839, 2015.

29. Wang M, Gao Q, Chen Y, Li Z, Yue L and Cao Y: PAK4, a target of miR-9-5p, promotes cell proliferation and inhibits apoptosis in colorectal cancer. Cell Mol Biol Lett 24: 58, 2019.

30. Ahmed SM, Ramani PD, Wong SQR, Zhao X, Ivanyi-Nagy R, Leong TC, Chua C, Li Z, Hentze H, Tan IB, et al: The chromatin structuring protein HMGA2 influences human subtelomere stability and cancer chemosensitivity. PLoS One 14: e0215696, 2019.

31. Dangi-Garimella S, Sahai V, Ebine K, Kumar K and Munshi HG: Three-dimensional collagen I promotes gemcitabine resistance in vitro in pancreatic cancer cells through HMGA2-dependent histone acetyltransferase expression. PLoS One 8: e64566, 2013.

32. Zhao X, Peter S, Dröge P and Yan J: Oncofetal HMGA2 effectively curbs unconstrained (+) and (-) DNA supercoiling. Sci Rep 7: 8440, 2017.

33. Tsavaris N, Lazaris A, Kosmas C, Gouveris P, Kavantzas N, Kopterides P, Papathomas T, Agrogiannis G, Zorzos H, Kyriakou V and Patsouris E: Topoisomerase I and IIalpha protein expression in primary colorectal cancer and recurrences following 5-fluorouracil-based adjuvant chemotherapy. Cancer Chemother Pharmacol 64: 391-398, 2009.

This work is licensed under a Creative Commons Attribution-NonCommercial-NoDerivatives 4.0 International (CC BY-NC-ND 4.0) License. 